\title{
MAC Protocol Design and Optimization for Multi-Hop Ultra-Wideband Networks
}

\author{
Lin X. Cai, Lin Cai, Member, IEEE, Xuemin (Sherman) Shen, Fellow, IEEE, Jon W. Mark, Life Fellow, IEEE, \\ and Qian Zhang, Senior Member, IEEE
}

\begin{abstract}
Ultra-wideband (UWB) communication is a promising enabling technology for future broadband wireless services. A simple, scalable, distributed, efficient medium access control (MAC) protocol is of critical importance to utilize the large bandwidth UWB channels and enable numerous new applications and services cost-effectively. In this paper, by investigating the characteristics of UWB communications, we propose a Distributed, EXclusive region (DEX) based MAC protocol. The proposed DEX protocol capitalizes on the spatial multiplexing gain of UWB networks by reserving exclusive regions (ER) surrounding the sender and receiver for data and acknowledgment (ACK) transmissions, so that users can efficiently and fairly share network resources in a distributed and asynchronous manner. We further quantify the network performance bounds and derive the optimal ER size to maximize the expected network transport throughput for a dense, multi-hop UWB network. Extensive simulation results demonstrate the efficiency and effectiveness of the DEX protocol. This work explores how to effectively utilize the wireless spatial capacity of distributed, multi-hop wireless networks by optimizing protocol parameters, instead of depending on more complicated control messages.
\end{abstract}

Index Terms-Distributed MAC protocol, multi-hop, UWB, distributed exclusive region.

\section{INTRODUCTION}

$\mathbf{U}$ LTRA-WIDEBAND (UWB) communications can achieve up to Gbps data rate with a transmission range of a few $(\leq 10)$ meters. Ubiquitous wireless access at a high data rate $(>100 \mathrm{Mbps})$ are possible using multi-hop small-range UWB transmissions. As synchronization and scheduling are difficult and costly in multi-hop UWB networks, it is desirable to have a simple, scalable, robust MAC protocol that allows users to efficiently utilize wireless resources in a distributed and asynchronous manner. The IEEE 802.11 distributed coordination function (DCF) has been overwhelmingly successful due to its flexibility, robustness, and simplicity. However, the efficiency of IEEE 802.11 DCF protocol in multi-hop wireless networks is far from ideal.

Manuscript received February 1, 2008; revised October 23, 2008 and February 18, 2009; accepted April 19, 2009. The associate editor coordinating the review of this paper and approving it for publication was Y. J. Zhang.

L. X. Cai, X. Shen, and J. W. Mark are with the Department of Electrical and Computer Engineering, University of Waterloo, Waterloo, ON N2L 3G1, Canada (e-mail: xshen@bbcr.uwaterloo.ca).

L. Cai is with the Department of Electrical and Computer Engineering, University of Victoria, BC V8W 3P6, Canada.

Q. Zhang is with the Department of Computer Science, Hong Kong University of Science and Technology, Hong Kong, China.

The research was supported in part by grants from Natural Sciences and Engineering Research Council of Canada (NSERC), RGC (CERG 622508, N_HKUST609/07), and the Foundation of Scientific and Technological Plan Project of Nansha, Guangzhou, China.

Digital Object Identifier 10.1109/TWC.2009.080155
In a dense multi-hop network, the throughput starvation and unfairness problems become severe due to the high contention level, which lead to unsatisfactory user experiences. Thus, the 802.11 DCF protocol cannot be directly used in dense multi-hop UWB networks. On the other hand, because of the stringent power limit of UWB imposed by the FCC regulations, power adaptation for UWB transmissions is generally not feasible; but rate adaptation is supported in all UWB PHY proposals [1], [2], [3], which should be considered for MAC protocol design. Also, UWB devices have very good ranging capability, which can be utilized by MAC protocols.

Recognizing the challenges and opportunities with UWB communications, in this paper, we propose a Distributed, EXclusive region (DEX) based MAC protocol, which is compatible with the existing 802.11 DCF protocol. Without relying on complicated control messages, we propose to optimize the main protocol parameters to enhance the protocol performance for UWB networks. Compared with the legacy 802.11 DCF protocol, DEX not only improves the transmission efficiency and network transport throughput, but also enhances fairness, because it effectively exploits the spatial reuse in dense UWB networks.

Spatial reuse has been well investigated in infrastructurebased cellular systems. It is well known that a higher spatial multiplexing gain can be achieved by using smaller cells at the cost of increased system management and control complexity (e.g., handoff overheads). Here, we investigate spatial multiplexing gain in a distributed multi-hop UWB network. In the proposed DEX protocol, a sender/receiver pair will use request-to-send/clear-to-send (RTS/CTS) messages to reserve smaller spatial areas, namely exclusive regions (ERs), for transmissions. By using the precise ranging capability of UWB devices, a node can decide whether it is within the ERs of the ongoing transmissions [4]. If yes, it will refrain from transmitting concurrently with the ongoing ones, and vice versa. Since only flows within the smaller ER compete with each other for channel access, more flows can transmit concurrently, and the throughput starvation and unfairness problems can be alleviated. We further derive the optimal ER size, which is generally much smaller than the carrier sensing region, such that the expected wireless network transport throughput can be maximized using the DEX MAC protocol.

The main contributions of this paper are three-fold. First, we propose an efficient distributed MAC protocol for a dense, multi-hop, large-scale UWB network. Second, we systematically analyze the performance of the proposed protocol. The 
TABLE I

NOTATIONS

\begin{tabular}{|c|l|}
\hline Symbol & Description \\
\hline \hline$C T$ & concurrent transmissions \\
$d\left(s_{j}, r_{i}\right)$ & distance between sender of flow $j$ and receiver of flow $i$ \\
$g\left(s_{i}, r_{i}\right)$ & channel gain for flow $i$ \\
$g\left(s_{j}, r_{i}\right)$ & channel gain between sender of flow $j$ and receiver of \\
& flow $i$ \\
$G_{0}$ & cross correlation between two flows \\
$I_{i}\left(s_{j}\right)$ & interference power from sender $j$ to receiver $i$ \\
$N_{0}$ & background noise power \\
$r_{i}$ & receiver of flow $i$ \\
$R(i)$ & transmission data rate of flow $i$ \\
$s_{i}$ & sender of flow $i$ \\
$P\left(r_{i}\right)$ & received power at receiver of flow $i$ \\
$P\left(s_{i}\right)$ & transmission power by sender of flow $i$ \\
$W$ & signal bandwidth \\
\hline
\end{tabular}

analytical framework can be applied to other distributed MAC protocols. Third, we further investigate how to appropriately set the protocol parameters to maximize the expected network transport throughput of a randomly and densely deployed UWB network. Extensive simulations are performed to verify the accuracy of the analysis and demonstrate the effectiveness and efficiency of the proposed protocol.

The rest of the paper is organized as follows. The system model is presented in Section II. In Section III, we propose the distributed MAC protocol, DEX, analyze its performance, and derive the network performance bounds. We then propose a method to optimize the ER size to maximize the expected network throughput. Simulation results are given in Section IV. Section V discusses related works, and concluding remarks and future research issues are given in Section VI.

\section{System Model}

Due to the stringent transmission power limit, normally the UWB transmission power level cannot be adjusted. Instead, the sender can adjust the transmission data rate according to the received signal-to-interference-plus-noise ratio (SINR). Because of the power constraint and the wide bandwidth in the UWB system, spreading technologies in both the time domain and the frequency domain are used [1], [3]. In a distributed network, different flows can use different spreading codes to reduce the mutual interference level among concurrent transmissions. Let $G_{0}$ denote the cross correlation between two concurrent transmissions using different (pseudo-random) spreading codes. Other traffic and channel parameters are tabulated in TABLE I for easy reference. In the system model, $G_{0}$ is assumed constant. The received SINR of flow $i$ is given by

$$
\operatorname{SINR}(i)=\frac{P\left(s_{i}\right) g\left(s_{i}, r_{i}\right)}{N_{0}+\sum_{j \neq i} P\left(s_{j}\right) g\left(s_{j}, r_{i}\right) G_{0}}
$$

In an additive white Gaussian noise (AWGN) channel, the data rate is upper bounded by the channel capacity $C=$ $W \log _{2}(S I N R+1)$ [5]. With fine-granularity rate adaptation technologies, the achievable link data rate is approximately given by

$$
R(i) \approx \eta W \log _{2}(S I N R(i)+1) \text { bps, }
$$

where $\eta$ is a system coefficient related to the efficiency of the transceiver design.
Due to the limited transmission range of UWB, multi-hop relay is necessary and favorable. To evaluate the protocol performance fairly in a multi-hop wireless network, transport throughput is usually used for performance evaluation, which is defined as the product of the throughput and the distance over which the information is being transferred. For MAC protocol evaluation, we focus on the transport throughput over each individual hop, i.e., the product of link throughput and distance. We will also investigate the delay and fairness (in terms of transport throughput) performance of the proposed protocol.

\section{DEX Protocol Design and Performance ANALYSIS}

Our protocol design principle is to choose simple and, ideally, existing building blocks for easy implementation, and to fine tune the protocol parameters for better performance. In this section, we introduce the proposed DEX-based MAC protocol, analyze its performance, and propose a method to optimize the protocol parameter.

\section{A. DEX Protocol}

Consider the employment of spreading codes for multiple access in which all nodes share a pool of spreading codes, numbered $1,2, \ldots, n$. One common spreading code is chosen for control message exchange, e.g., for RTS and CTS frames. Each node maintains a code table to record all the spreading codes used by the ongoing neighboring transmissions. The procedures to choose codes and initiate transmissions at the sender and receiver sides are given in Algorithm 1 and Algorithm 2, respectively.

Each node maintains a code table and a network allocation vector (NAV). The initial contention window size equals the minimum window size, i.e., $C W=C W_{\min }$, and the node sets its retry counter to 0 .

If node $A$ receives data from the upper layer for transmission to node $B, A$ will use a hash function to obtain a spreading code: $X=\operatorname{Hash}(A+B)$ for the transmission, where $A$ and $B$ used in the hash function are related to their MAC addresses. $A$ starts channel sensing when its $N A V$ reaches zero. If the channel is sensed idle for a backoff interframe space $(B I F S), A$ transmits an RTS frame to $B$, including the chosen code $X$ and the transmission time $T_{2}=R T S+S I F S+C T S+S I F S+D A T A+A C K$. Otherwise, $A$ enters a backoff procedure and sets a backoff counter $(B C)$ uniformly distributed over $[0, C W)$ for the first transmission attempt and $A$ freezes its $B C$ until the channel is sensed idle for $B I F S$.

If the channel is sensed busy but $A$ has not successfully received an RTS or CTS, $A$ needs to continue channel sensing till the channel is idle for BIFS. If $A$ overhears an RTS or CTS frame from another transmission $f_{i}, A$ checks the ER condition: 1) if either the transmitter or the receiver of $f_{i}$ is in $A$ 's ER region, $A$ should postpone its own transmission until the ongoing transmission $f_{i}$ completes, and $A$ sets its $N A V$ according to $T_{2} ; 2$ ) if $A$ is outside the ER of $f_{i}, A$ only needs to wait until RTS times out and sets $N A V$ according to $T_{1}=R T S+S I F S+C T S$. An example of $N A V$ setting is shown in Fig. 1, where $A$ and $B$ exchange RTS and CTS 


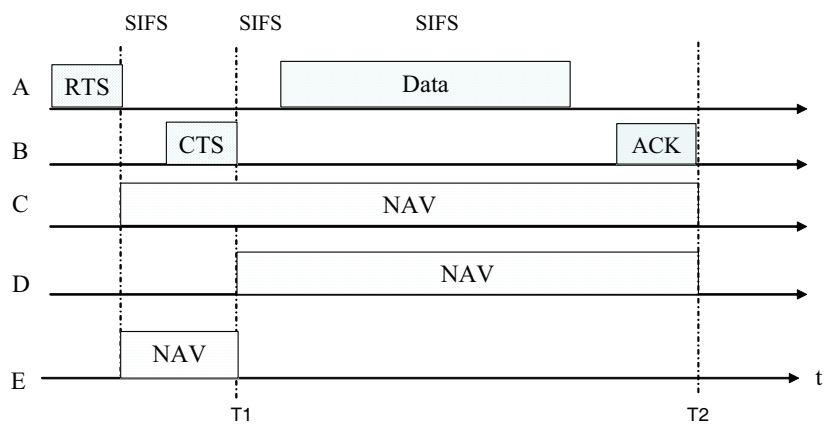

Fig. 1. Network allocation vector update.

messages, $C$ and $D$ are neighbors within the ER of flow $A B$, respectively, and $E$ is another neighbor outside the ER of flow $A B$. Since $A$ is outside the ER of $f_{i}$ and concurrent transmission is allowed, $A$ adds the spreading code used by $f_{i}$ in its code table and assures that its own code $X$ does not conflict with any record in its code table. If code collision occurs, $A$ can hash again till there is no code collision. Each record in the code table is associated with a time to live (TTL) parameter and will be removed from the table if TTL expires.

If $A$ successfully receives a CTS from $B$ after an interval SIFS, implying that $B$ is available for the transmission using the spreading code $X, A$ starts to transmit data to $B$ at a rate of $R(i)$ after a SIFS. For implementation simplicity, the rate $R(i)$ is not determined based on the measurement of the instantaneous interference and noise level of the tagged transmission, but on the worst case scenario that assumes the maximum number of dominant interferers. Therefore, DEX is robust against interference from neighborhood asynchronous transmissions. The detail derivation of $R(i)$ is presented in Sec. III-B. If no CTS is received successfully, implying that $B$ is not available at this moment to receive data using code $X$, $A$ will enter the backoff stage and retransmit thereafter, until the retransmission limit $m$ is reached. The backoff procedure in DEX is the same as that in IEEE 802.11. Each time $A$ retransmits RTS, it will also choose a different code $X$ by repeating hash functions because the code it chose previously may not be acceptable for use at $B$, (although the probability of code collision at $B$ is very low).

To further improve the protocol efficiency, a transmission opportunity (TXOP) is employed, i.e., a time duration $T$ is reserved in each RTS/CTS that a transmitter can transmit a burst of data frames during $T$. The longer the $T$, the better resource utilization will be, because less overhead is involved in each transmission. But a longer $T$ leads to a larger access delay. Therefore, $T$ should be chosen appropriately so that the access delay is tolerable for other flows in the ER region. On the other hand, a smaller ER region allows for more concurrent transmissions, which reduces the access delay of each flow. Thus, it is possible to choose a larger $T$ for DEX and still well maintain the desired delay and fairness performance.

At the receiver side, $B$ is ready for channel sensing or receiving only if its $N A V=0$. Whenever $B$ overhears an RTS or CTS frame from its neighboring node, $B$ will update its $N A V$ and code table in the same way as sender $A$ does. Upon successfully receiving an RTS from $A, B$ sends back a CTS if $X$ does not conflict with any record in $B$ 's code table and the channel is idle for a SIFS period. Otherwise, $B$ keeps silent and $A$ may retransmit an RTS and choose another code after the RTS timeout.

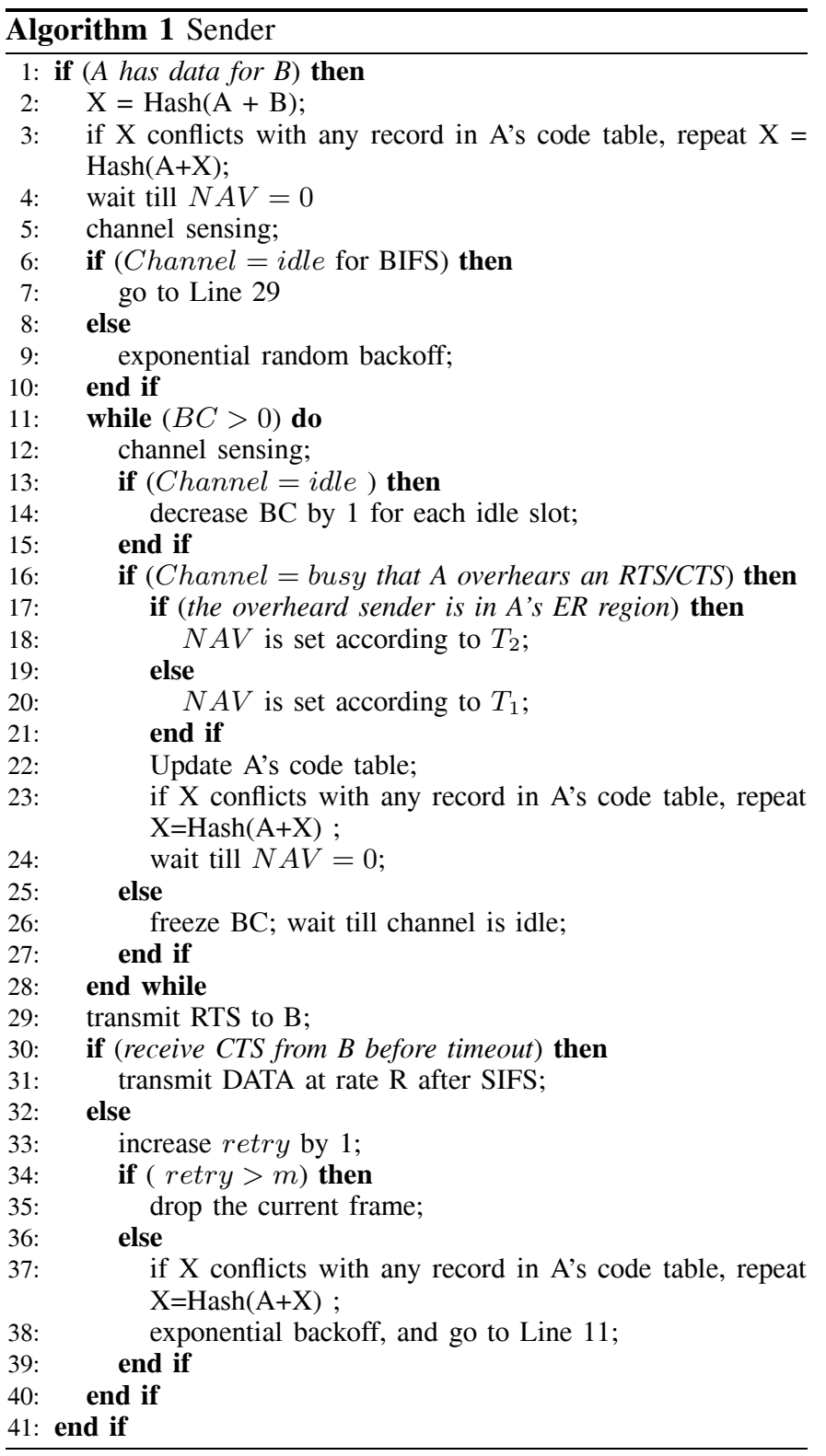

\section{B. Protocol Performance and Parameter Setting}

In this subsection, we first prove that the DEX protocol is correct, i.e., for each pair of nodes that successfully exchanges RTS/CTS messages, their data transmissions can be collision-free. Here, collision-free means that the interference from other concurrent transmissions is less than the tolerable threshold. We also study the performance bounds of a dense multi-hop UWB network and propose a method to choose ER size appropriately towards the maximum network throughput in a randomly deployed network.

To evaluate the network performance, we use the $\log$ distance path loss model for signal loss in an indoor radio propagation channel, which is given by

$$
P L(d)=P L_{0}+10 \alpha \log _{10}\left(d / d_{r e f}\right),
$$



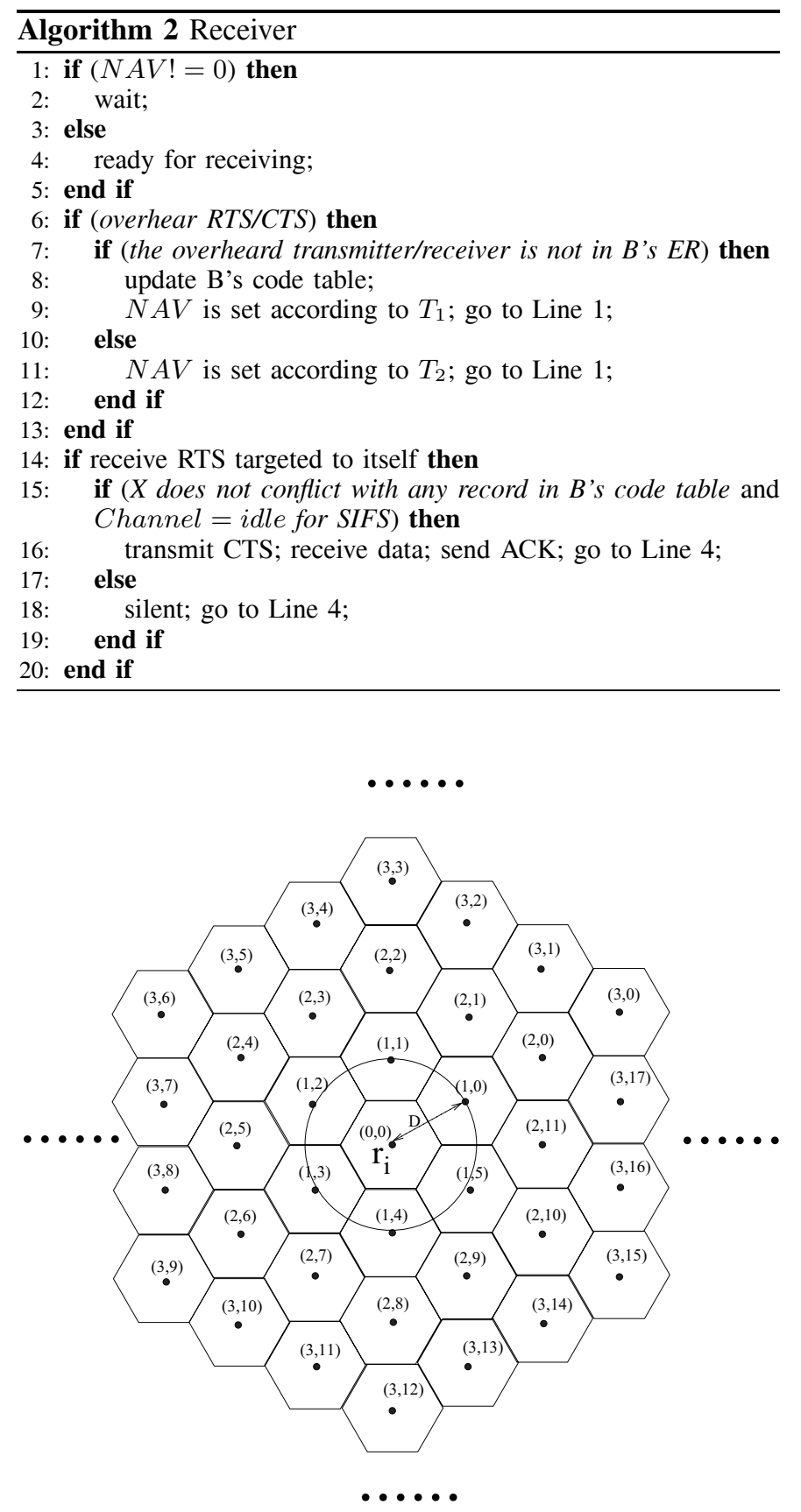

Fig. 2. Worst-case scenario with the maximum interference.

where $P L(d)(\mathrm{dB})$ is the total path loss at distance $d, P L_{0}$ is the path loss at the reference distance $d_{r e f}=1 \mathrm{~m}, \alpha$ is the path loss exponent. Under this channel model, the received SINR of flow $i$ is given by

$$
\operatorname{SINR}(i)=\frac{P\left(s_{i}\right) d\left(s_{i}, r_{i}\right)^{-\alpha}}{N_{0}+\sum_{j \neq i} P\left(s_{j}\right) d\left(s_{j}, r_{i}\right)^{-\alpha} G_{0}},
$$

where $P\left(s_{i}\right)$ is the transmission power of the sender of flow $i, d\left(s_{j}, r_{i}\right)$ is the distance between the sender of flow $j$ and the receiver of flow $i$.

1) Protocol Correctness: Considering a network with random topology and user deployment, we first consider the maximum amount of interference generated by concurrent transmissions from other nodes to the tagged receiver $r_{i}$.

The highest interference level is related to the "circle packing" problem. Each sender is located in the mid-point of an ER which is a circle. The maximum interference occurs when the non-overlapping circles are packed in the plane with highest density, which has been proved to be the hexagonal packing. Thus, the maximum interference to the receiver $r_{i}$ occurs when all interferers are located in the center of each hexagon cell around $r_{i}$, as shown in Fig. 2, i.e., there are 6 interferers located in the first tier of cells of $r_{i}$, and $6 k$ interferers located in the $k$-th tier of cells. The distance from the $k$-th tier interferers to $r_{i}$ is no less than $\sqrt{3} k D / 2$. Thus, the total interference to $r_{i}, I_{r}$, is bounded by

$$
\begin{aligned}
I_{r, D} & <\sum_{k=1}^{\infty} 6 P G_{0}(\sqrt{3} D / 2)^{-\alpha}(k)^{1-\alpha} \\
& =6 P G_{0}(\sqrt{3} D / 2)^{-\alpha} \zeta(\alpha-1) .
\end{aligned}
$$

The above Riemann Zeta-function, $\zeta(\alpha-1)$, converges iff $\alpha>2$. Therefore, if the path loss $\alpha$ is a constant not larger than 2 , an infinite coverage area cannot allow an infinite number of concurrent transmissions, and vice versa. Fortunately, empirical evidences from experimental field studies suggest that while path loss exponent near the transmitter is likely to be 2 , at large distance, it is larger than 2 , and the received power level even decays exponentially with distance if the distance is quite large. Therefore, the interference to a tagged user can be well bounded. With a random network setting, it is practical to assume that the maximum interference to $r_{i}, I_{r, D}$, is $6 P G_{0} D^{-\alpha}$, because (a) the number of first tier interferers is less than six almost surely, (b) the number of interferers is finite, and (c) the path loss exponent is large for high dense wireless networks, so the value of $\zeta(\alpha-1)$ is close to one.

Now, we can prove that the sender and receiver which successfully exchange RTS/CTS can successfully transmit without being interrupted by other users at rate $R(i)=$ $\eta W \log _{2}\left(P\left(r_{i}\right) /\left[N+I_{r, D}\right]+1\right)$. First, since the RTS/CTS of the pair has been successfully exchanged, all other nodes within their ER will not interrupt the tagged transmission. Second, the actual SINR should be larger than $I_{r, D}$. Thus, the transmission can be successful because the data rate chosen by the pair is more conservative than the actual achievable one.

2) Hidden terminal and exposed terminal: We examine the hidden terminal and exposed terminal problems in multi-hop wireless networks. The hidden terminal problem exists for RTS transmissions. Since DEX allows concurrent transmissions and each pair of nodes can transmit data/ACK for a comparatively long time $T$ consecutively, the number of RTS messages exchanged is reduced, so the collisions due to hidden terminal are reduced. In addition, if we can set the carrier sensing range to be the sum of the transmission range and the interference range of RTS, we can eliminate hidden terminals.

Using 802.11 DCF, there are proposals to mitigate the hidden terminal problem, which usually leads to more severe exposed terminal problem. The nice feature of the proposed DEX protocol is that the reserved space by RTS/CTS is determined by the ER region, instead of the carrier sensing range, so it does not suffer from the exposed terminal problem as much as the IEEE 802.11 DCF protocol. Since the ER is much smaller than their carrier sensing regions, more flows 


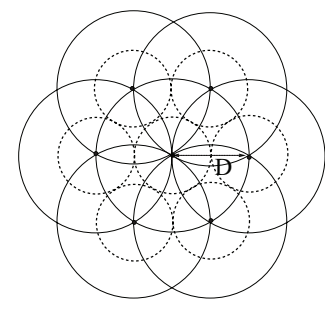

(a)

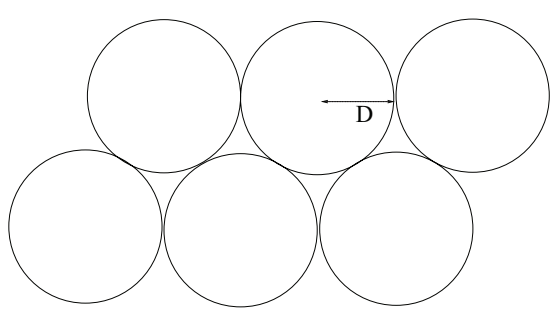

(b)
Fig. 3. (a) Circle packing problem; (c) circle covering problem.

can transmit concurrently, and the spatial multiplexing gain of DEX is higher than that of 802.11 DCF.

Another issue may result in performance degradation is that the RTS message in 802.11 DCF will notify all other nodes within the carrier sensing range of the sender to refrain from contention till the end of the transmission. If the receiver cannot send CTS because it is inside the carrier sensing range of some ongoing transmissions, the reservation of the RTS is totally wasted. In addition, if both the sender and the receiver of a flow need to compete with a large number of other nodes, the flow may starve due to the low possibilities that both the sender and the receiver can successfully access the channel. With DEX, since the ER is smaller than the carrier sensing region, the chance that the receiver cannot reply to the RTS is much lower, and the starvation problem can be alleviated.

3) Network Performance Bounds: We investigate the performance of the DEX protocol and derive the performance bounds. A node's ER is a circle centered at the node with radius $D . D$ is a key parameter, which affects the number of concurrent transmissions in an area and the interference level to a tagged user. Considering a dense network, we are interested in obtaining the optimal value of $D$ which can maximize the expected network transport throughput. To derive the throughput, we need to know the average number of concurrent transmissions (CTs), which is very difficult to obtain because it is sensitive to the network topology and the sequence of nodes initiating transmissions. Thus, we first obtain the theoretical upper bound and lower bound of the number of CTs in a dense network.

Lemma 1: In an area of $L \times L$, for a given ER with radius $D$, the upper bound of the number of CTs is $2 L^{2} /\left(D^{2} \sqrt{3}\right)$.

Proof: As shown in Fig. 3, in the extreme case that, for each flow, the ER region (the solid circles) of the sender and that of the receiver fully overlap, the maximum number of CTs is equivalent to the maximum number of circles with radius $D / 2$ (the dashed circles) that can be packed in the area. This is the classical circle packing problem. Toth proved that the hexagonal lattice is indeed the densest of all possible plane packings [6]. Accordingly, the maximum number of CTs is $2 L^{2} /\left(D^{2} \sqrt{3}\right)$.

Remark: In a sparse network, we can improve the network throughput by increasing the node density to enlarge the number of CTs. However, according to Lemma 1, once the node density is large enough to saturate the network, further increasing the node density cannot improve the network throughput, but only increase the competition levels of all nodes within the associated ER and results in severe collisions.

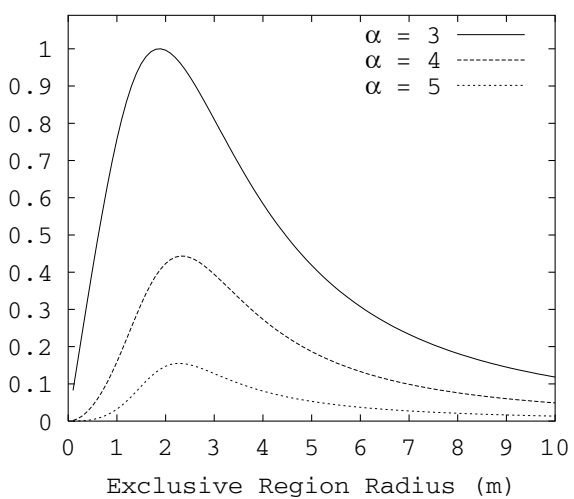

Fig. 4. Normalized expected network transport throughput.

The lower bound of the number of CTs in a sparse network can be as low as zero and it is not of our interest. In the following, we consider the lower bound of a saturated network. Wireless resources have three dimensions: time, frequency, and space. In a saturated network, we assume that if there is any unoccupied time/frequency/space to allow new collisionfree transmissions, some node will initiate a transmission. As one of the main concerns for MAC protocols is to control congestion in the link layer, performance study for saturated networks can provide important insights and guidelines.

Lemma 2: Given the ER with radius $D$ in a saturated network of size $L \times L$, the lower bound of the number of CTs is $L^{2} /\left(\sqrt{27} D^{2}\right)$.

Proof: We consider the extreme scenario that the ERs of all senders and receivers do not overlap with each other. For a given ER with radius $D$ and a saturated network of size $L \times L$, the lower bound of the number of CTs is equivalent to half of the minimal number of circles with radius $D$ that can cover the area. This is the classical circle covering problem. It has been derived that a lower bound for a covering using equivalent circles is $2 \pi / \sqrt{27}$ [7]. Thus, the minimal number of circles covering an area equals $(2 \pi / \sqrt{27})\left(L^{2} / \pi D^{2}\right)=$ $2 L^{2} /\left(\sqrt{27} D^{2}\right)$. Given that each flow has two non-overlapping circles, the minimal number of CTs in a saturated network is $L^{2} /\left(\sqrt{27} D^{2}\right)$.

4) Exclusive Region Size: From Lemmas 1 and 2, for a saturated network, the upper bound of the number of CTs is six times that of the lower bound, and both are proportional to $D^{-2}$. The distribution of the number of CTs in a random network is very difficult if not impossible to obtain. Nevertheless, the expected number of CTs should be proportional to $D^{-2}: \mathrm{E}[\mathrm{CT}]=\mathrm{k}_{1} / \mathrm{D}^{2}$, where $k_{1}$ is an unknown coefficient.

As the REX sender will use $6 P G_{0} D^{-\alpha}$ as the interference level to set the transmission rate, we then estimate the expected network transport throughput, as given by

$$
\frac{k_{1}}{D^{2}} \eta W E[d] \log _{2}\left(1+\frac{P E[d]^{-\alpha}}{N+6 P G_{0} D^{-\alpha}}\right),
$$

where $E[d]$ is the expected transmission distance. As the expected transport throughput is a non-linear function of $d$, using $E[d]$ to get the expected transport throughput is an approximation. Simulation results show that the above approximation is acceptable. Taking the derivative of (6), we can obtain the optimal $D$ value which maximizes the expected 
TABLE II

SYSTEM PARAMETERS

\begin{tabular}{|c|c||c|c|}
\hline$W$ & $500 \mathrm{MHz}$ & BIFS & $20 \mu \mathrm{s}$ \\
\hline$P\left(s_{i}\right)$ & $-41.3 \mathrm{dBm} / \mathrm{MHz}$ & SIFS & $10 \mu \mathrm{s}$ \\
\hline$N_{0}$ & $-114 \mathrm{dBm} / \mathrm{MHz}$ & a slot time & $20 \mu \mathrm{s}$ \\
\hline$\alpha$ & $2.5-6$ & $C W_{\min }$ & 31 \\
\hline$G_{0}$ & $0.01-1$ & $C W_{\max }$ & 1023 \\
\hline$\eta$ & 0.21 & maximum retry limit & 7 \\
\hline$d_{r e f}$ & $1 \mathrm{~m}$ & RTS/CTS & $20 \mu \mathrm{s}$ \\
\hline$P L_{0}$ & $43.9 \mathrm{~dB}$ & Transmission range & $10 \mathrm{~m}$ \\
\hline
\end{tabular}

TABLE III

Optimal ExCluSive REgION Size (ANALYSis)

\begin{tabular}{|c|c|c|c|}
\hline$\alpha$ & $G_{0}=0.01$ & $G_{0}=0.1$ & $G_{0}=1$ \\
\hline 3 & $1.87 \mathrm{~m}$ & $4.03 \mathrm{~m}$ & $8.69 \mathrm{~m}$ \\
\hline 4 & $2.34 \mathrm{~m}$ & $4.15 \mathrm{~m}$ & $7.39 \mathrm{~m}$ \\
\hline 5 & $2.28 \mathrm{~m}$ & $3.61 \mathrm{~m}$ & $5.72 \mathrm{~m}$ \\
\hline 6 & $2.11 \mathrm{~m}$ & $3.10 \mathrm{~m}$ & $4.55 \mathrm{~m}$ \\
\hline
\end{tabular}

network transport throughput.

Fig. 4 shows the normalized expected network transport throughput as a function of the exclusive region of radius $D$, with $G_{0}=0.01$, using the parameters listed in TABLE II. It is shown that the expected network transport throughput is a concave function of $D$, while fixing other parameters, including $P, W, N_{0}$, etc. The best value of $D$ can be determined when the maximum expected throughput is achieved. The analytical results of optimal $D$ under different parameter values of $\alpha$ and $G_{0}$ are listed in TABLE III. It is observed that the optimal $D$ becomes larger when $G_{0}$ increases, but changes less with $\alpha$. This is because the path loss exponent $\alpha$ affects both the received signal strength and the interference level and the corresponding SINR does not change much, while the cross correlation, $G_{0}$, determines the interference only (in the denominator of SINR). A greater $G_{0}$ means more serious interference among concurrent transmissions, and thus a larger $D$ is required to bound the total interference level to achieve high network throughput.

In practical, the value of $\alpha$ may not be accurately measured or estimated, so the value of $D$ may not be optimal. However, as shown in Fig. 4, the optimal values of $D$ for $\alpha=3,4,5$ only have small difference. Thus, even we under- or overestimate $\alpha$, the value of $D$ chosen by the DEX protocol can still be close to the optimal value.

\section{Simulations Results}

In this section, we evaluate the performance of the proposed DEX protocol in terms of transport throughput, fairness, and access delay, and compare it with that of the IEEE 802.11 DCF via simulations. We choose the IEEE 802.11 DCF protocol as benchmark since it is the most popular asynchronous MAC protocol widely adopted.

\section{A. Simulation Settings}

The simulated network is set up in a $20 \mathrm{~m} \times 20 \mathrm{~m}$ square room, which contains up to 100 active flows, with distinct senders and receivers uniformly distributed in the room. The simulation parameters are listed in TABLE II. The senders use the maximum transmission power and the transmission range is $10 \mathrm{~m}$. The background noise power is $4 \times 10^{-9} \mathrm{~mW}$ over

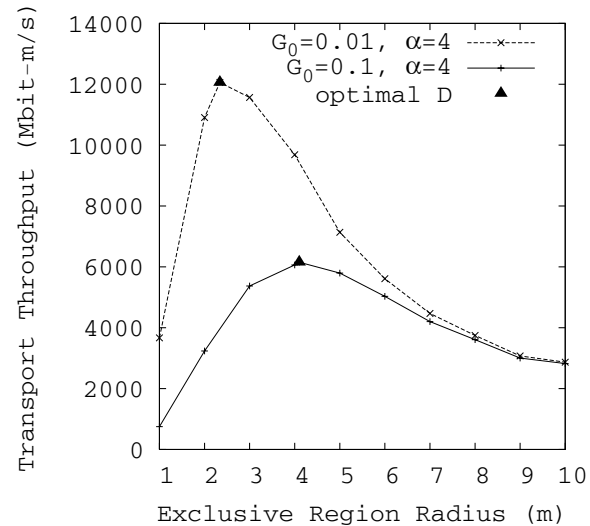

Fig. 5. Transport throughput vs ER radius.

$500 \mathrm{MHz}$ signal bandwidth [1]. All $N$ active flows contend for channel access in an asynchronous manner, with its initial arrival time uniformly chosen over $[0,32)$ time slots. The reference distance is set as $d_{r e f}=1 \mathrm{~m}$, and the path loss at $d_{r e f}$ is $43.9 \mathrm{~dB}$. Thus, the maximum data rate at $1 \mathrm{~m}$ is given by (2), i.e., $R=\eta W \log _{2}(1+S I N R)=1 \mathrm{Gbps}$. The achievable data rate decreases with distance $d$, e.g., given the path loss exponent $\alpha=4$, the received $S I N R$ degrades from $28.8 \mathrm{~dB}$ at $d_{\text {ref }}=1 \mathrm{~m}$ to $16.76 \mathrm{~dB}$ at $d=2 \mathrm{~m}$ and the achievable data rate at $2 \mathrm{~m}$ decreases to $R=585 \mathrm{Mbps}$. To eliminate the warming-up effects, the simulation data are collected from $10 \mathrm{~s}$ to $60 \mathrm{~s}$. We repeat each simulation 10 times with different random seeds and calculate the average.

\section{B. Transport Throughput}

The transport throughput of a dense network with 40 flows using the DEX protocol is shown in Fig. 5. The data transmission time is $T=10 \mathrm{~ms}$. When the ER radius $D$ is very small, more flows are likely to be outside of each other's ER to transmit concurrently; however, a smaller $D$ results in a higher interference level that decreases the data transmission rate. It is observed in Fig. 5 that the total transport throughput of the network is maximized if the value of $D$ is close to the optimal value obtained from the analysis. When the cross correlation $G_{0}$ is larger, the interference level among concurrent flows becomes more serious so we should enlarge the value of $D$ accordingly. Simulation results validate the accuracy of our analysis, which demonstrate the significant spatial multiplexing gain achieved by the proposed DEX protocol.

Another observation from Fig. 5 is that, if the value of $D$ is slightly different from the optimal, the throughput is slightly below the highest one. Combining this observation with the results shown in Fig. 4, we can claim that, even if the value of $\alpha$ is not accurately obtained, the protocol performance of DEX will not degrade significantly.

We then investigate the network transport throughput under various network densities, and compare the performance of the DEX protocol (with ER radius $D=4.15 \mathrm{~m}, G_{0}=0.1$, $T=10 \mathrm{~ms}$ and $\alpha=4$ ) with that of IEEE $802.11 \mathrm{DCF}$ (with carrier sensing range of $10 \mathrm{~m}$ ) in Fig. 6. When there are only 10 flows in a $20 \mathrm{~m} \times 20 \mathrm{~m}$ square room, the network is relatively sparse, and the transport throughput of 


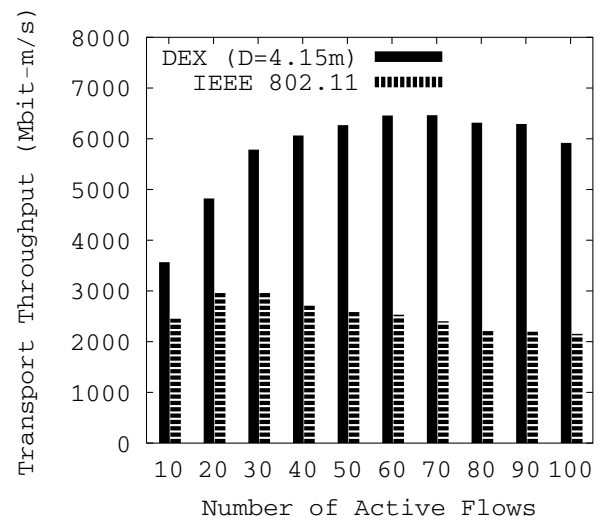

Fig. 6. Transport throughput comparison of different protocols.

the DEX protocol is 1.45 times that of IEEE 802.11 DCF. When the number of flows exceeds 30 , the network throughput of IEEE 802.11 DCF decreases due to serious collisions among the competing flows; while with the proposed DEX, more concurrent transmissions are allowed when the number of active flows increases. The achieved transport throughput of DEX is around 2 times that of IEEE 802.11 DCF with 30 active flows, and 2.7 times with 70 active flows. It is also shown in Fig. 6 that the network transport throughput of DEX increases w.r.t. the network density until the number of active flows in the network exceeds 70 , when serious collisions degrade the network performance. In all cases, the proposed DEX significantly outperforms IEEE 802.11 DCF by aggressively exploiting spatial reuse opportunities, and it is more suitable for a dense multi-hop UWB network.

We further study the impact of data transmission time $T$ on both protocols. In the DEX protocol, we use the optimal ER radius $D=4.15 \mathrm{~m}$ for $G_{0}=0.1$ and $\alpha=4$. It is observed in Fig. 7 that the transport throughput of 40 active flows increases with the data transmission time $T$ in both DEX and IEEE 802.11. With a larger $T$, the protocol overheads, including RTS/CTS, backoff time, interframe space, etc., become relatively smaller, and more flows can transmit concurrently to achieve a higher spatial multiplexing gain with DEX. As shown in Fig. 7, the ratio of the achieved transport throughput using DEX at optimal $D$ to that of IEEE 802.11 increases from 1.6 for $T=0.5 \mathrm{~ms}$ to 2.29 for $T=10 \mathrm{~ms}$. The proposed DEX always outperforms IEEE 802.11 w.r.t. various $T$ values. It is worth noting that a large value of $T$ is preferable for network throughput, but it will result in unfairness problem and longer access delays for other flows in the same contention region. In the following subsections, we investigate the fairness and access delays.

\section{Fairness}

Fairness is evaluated using Jain's fairness index [8], in terms of the network transport throughput. We first compare the fairness performance of DEX under various $D$ values with that of IEEE 802.11 DCF in Fig. 8. It is well known that the 802.11 DCF based MAC exhibits serious unfairness among competing flows in a multi-hop environment. Some "lucky" flows are more likely to access the channel, while other "unlucky" ones may suffer from complete throughput

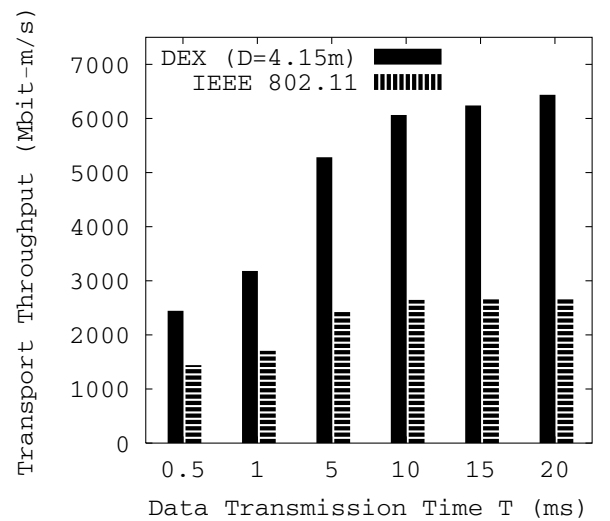

Fig. 7. Transport throughput vs data transmission time $T$.

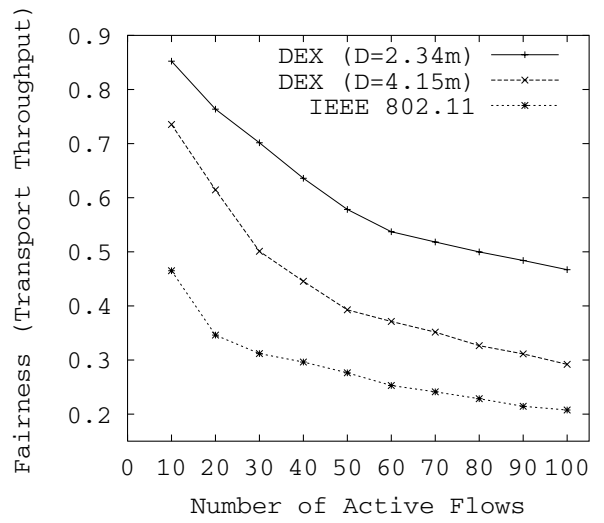

Fig. 8. Fairness (Transport throughput) comparison of different protocols.

starvation. Starvation phenomenon becomes more severe in a denser network. As shown in Fig. 8, the fairness performance of both protocols decreases when the number of active flows increases. However, the proposed DEX with smaller ER radius $D$ achieves better fairness than the 802.11 DCF. This is because smaller ER region can effectively reduce the flow starvation by reducing the number of competing flows, and accordingly improve the fairness performance.

The impact of $T$ on fairness is shown in Fig. 9. We consider 40 active flows in the room. With a larger value of $T$, all neighbors within the ER of the tagged sender and receiver have to postpone their transmissions for a longer duration, and thus they are more likely to starve, especially when the ER radius is large and there are many competing flows in the neighborhood. As shown in Fig. 9, the fairness performance degrades significantly when the ER radius $D$ and data transmission time $T$ increase.

\section{Delay Outage Ratio}

Access delay is another important performance metric for evaluating a MAC protocol. We define the delay outage ratio as the ratio of the number of attempts with access delay exceeding the delay threshold to the total number of attempts. We set the delay threshold to $150 \mathrm{~ms}, G_{0}=0.1$ and $\alpha=4$. As shown in Fig. 10, the delay outage ratio increases with the number of active flows because more collisions among competing flows result in more backoff and thus longer 


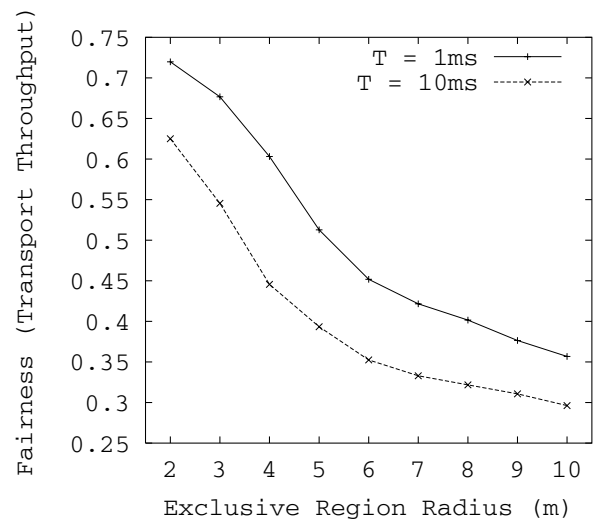

Fig. 9. Fairness (transport throughput) under various ER radius.

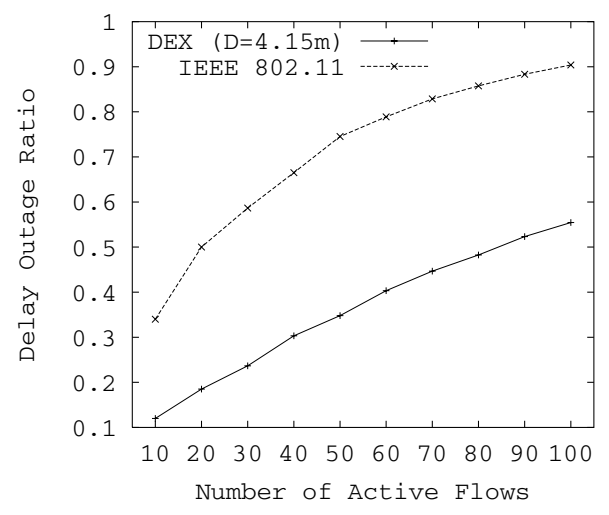

Fig. 10. Delay outage ratio comparison of different protocols.

access delays. However, the proposed DEX allows for more concurrent transmissions, so the average access delay of each flow is reduced when the ER radius is smaller.

For choosing the value of $T$, there is a tradeoff between fairness/delay and transport throughput. We can choose the maximum value of $T$, under the constraints that the corresponding fairness index is above certain threshold and the delay outage ratio is below certain threshold. Since the ER of DEX is much smaller than the carrier sensing region of 802.11 DCF, DEX can use a larger value of $T$ for higher throughput and still maintain a desirable delay and fairness performance.

\section{Related Work and Existing Protocols}

Generally, MAC protocols can be classified into two categories: centralized and distributed MAC. Extensive research has been conducted on developing efficient centralized MAC protocols for wireless networks, with dedicated or randomly chosen coordinators [9]. A centralized MAC protocol usually provides more reliable and predictable services than distributed MAC at the expense of control overheads. The order-optimal wireless capacity with ideal scheduling in the MAC layer was investigated in [10]. The optimal throughput and delay tradeoff in static wireless networks was studied in [11]. It was claimed in [12] that in wideband systems, it is optimal to have an ER around the receiver. Based on the ER concept proposed in [12], the capacity of the infrastructure-

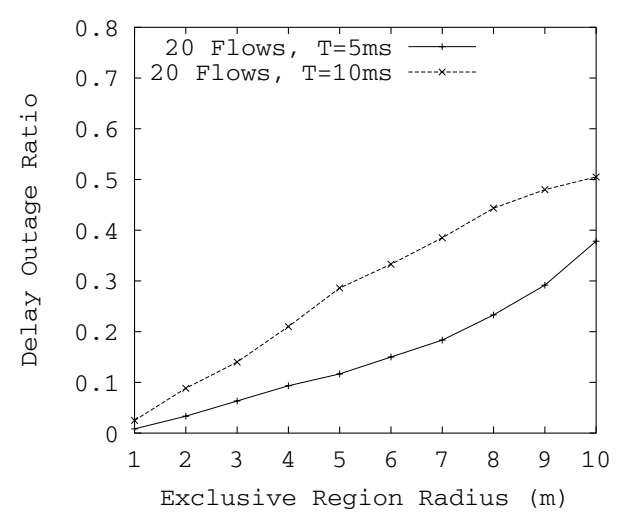

Fig. 11. Delay outage ratio vs ER radius.

based UWB networks with the dedicated resource allocation scheme and the random access MAC were studied in [13] and [14], respectively. The optimal resource management was formulated as a utility maximization problem in [15], based on the global user information at the central controller.

However, a centralized solution may not be desirable for large-scale, multi-hop wireless networks for the following reasons: a) Centralized schemes normally have significant communication and computational overheads. With high data rate (up to Gbps) UWB communication technologies, the transmission time is usually on the order of $\mu$ s, so any packetlevel scheduler with complexity more than $O(1)$ becomes less desirable. On the other hand, the traffic of many applications, e.g., data and video traffic, are bursty in nature, thus it is difficult to reserve an appropriate amount of resources for these traffic flows; b) tight synchronization among devices is costly, especially in dense UWB networks; c) centralized architecture is less scalable, and it may suffer the singlepoint-of-failure problem; d) when a hierarchical structure is used to divide the entire network into multiple small piconets, coordination among piconets is not an easy task.

The WiMedia Alliance has launched PHY and MAC layer specifications based on the Multi-band Orthogonal Frequency Division Multiplexing (MB-OFDM) UWB technology [3]. The WiMedia MAC specification uses a combination of CSMA and TDMA mechanisms to provide a certain level of quality of service for isochronous traffic in a distributed manner. In [16], a contention based distributed algorithm, RCAMA, is proposed, using a physical interference model. However, both RCAMA and WiMedia MAC are based on a time-slot frame structure, which still requires tight synchronization. The maximum throughput region attained by a distributed scheduling strategy under arbitrary network topology and interference models was given in [17]. A distributed maximal matching scheduling strategy was presented in [18] to guarantee a certain fraction of the optimal throughput region. In [19], a distributed greedy scheduling scheme based on a more general interference model was proposed, and a lower bound on the capacity region was also investigated. However, to guarantee the throughput performance, the schemes in [17], [18], [19] may require many rounds of computation and control message exchanges, and thus are not scalable because the overheads increase with the network size.

These works mainly focused on proposing new protocols 
or algorithms with more complicated control messages and computations to improve the resource utilization. In addition, the rate adaptive characteristics of the UWB communication technologies were not considered. A joint PHY/MAC architecture for impulse-based time-hopping UWB was proposed in [20], considering power control, rate adaptation, and mutual exclusive region. The approach mainly focused on effective physical layer modulation schemes to cancel the interfering energy, and effective MAC protocol design still remains an open issue.

On the other hand, several recent works have been proposed to adaptively adjust the carrier sensing range of the transmitters to improve the spatial reuse performance of IEEE 802.11 DCF [21], [22], [23], [24], [25]. In [22], the relationship between transmission power and the carrier sense threshold was studied, assuming a perfect MAC protocol that all communication channels are fully utilized. The impact of the carrier sensing threshold on the network capacity was investigated in [23]. The optimal carrier sensing threshold that maximizes spatial reuse for several regular topologies was obtained in [24]. It was found in [25] that the optimal carrier sensing range of 802.11 DCF MAC should consider the tradeoff between the spatial reuse and the packet collision probability, and that an optimal carrier sensing range can be obtained based on a reward formulation. However, all the previous works use a simple collision model (A collision occurs if two or more stations within their transmission ranges transmit simultaneously.) in a WLAN environment, where the signal and interference levels are much higher than those in UWB networks. Due to the stringent power emission regulation and the wide bandwidth of UWB communications, spreading technologies are usually employed to allow multiple concurrent transmissions [1], [3]. Thus, the simple collision model used in WLANs does not hold in UWB systems. In addition, adjusting the carrier sensing range of the transmitters can only reduce possible collisions among those transmitters within their carrier sensing ranges, but cannot guarantee successful receptions at the receivers. Therefore, instead of adjusting sensing ranges around the transmitters, we define exclusive regions around the receivers to assure that the ongoing transmission to the tagged receiver will not be interrupted by other interferers. To the best of our knowledge, little work has been done for asynchronous distributed MAC design and optimization for multi-hop UWB wireless networks, considering the characteristics of UWB communication technologies. Thus motivated, we propose the DEX MAC protocol to efficiently exploit the spatial capacity of multi-hop UWB networks.

\section{CONCLUSiOn AND Future WORK}

In this paper, we have proposed a distributed MAC protocol, referred to as DEX, for multi-hop UWB networks. With the derived optimal ER size, DEX can effectively and efficiently utilize network resources in an asynchronous and distributed manner. Our work suggests a new direction of future MAC protocol design for high data rate, dense networks. Instead of depending on more complicated control messages, we have investigated the protocol parameters and improve the protocol performance significantly by fine tuning them. An important future research issue is to study the performance of the proposed DEX protocol in the presence of fast fading and shadowing in the UWB channel. Another possible way to implement the DEX protocol is to use the average received signal strength instead of the geometry distance to determine the exclusive region. In this case, how to promptly obtain accurate signal strength of UWB communications requires further investigation.

\section{REFERENCES}

[1] I. . W. H. R. A. P. T. G. 3a, "DS-UWB physical layer proposal," Jan. 2005.

[2] — "MultiBand OFDM physical layer proposal," Sept. 2004.

[3] S. ECMA-368, "High rate ultra wideband PHY and MAC standard, 2nd ed." Dec. 2007. [Online]. Available: http://www.ecmainternational.org/publications/standards/Ecma-368.htm

[4] R. Cardinali, L. D. Nardis, M. G. D. Benedetto, and P. Lombardo, "UWB ranging accuracy in high- and low-data-rate applications," IEEE Trans. Microwave Theory and Techniques, vol. 54, no. 4, pp. 1865-1875, Apr. 2006.

[5] C. E. Shannon, "A mathematical theory of communication," Bell System Technical J., vol. 27, pp. 379-423, July 1948.

[6] H. SteinhausM, Mathematical Snapshots (3rd ed.). New York: Dover, 1999.

[7] R. Williams, "Circle coverings," Geometrical Foundation of Natural Structure: A Source Book of Design, pp. 51-52, 1979.

[8] R. Jain, A. Durresi, and G. Babic, "Throughput fairness index: an explanation," ATM Forum Document Number: ATM Forum/990045, Tech. Rep., Feb. 2005.

[9] X. Shen, W. Zhuang, H. Jiang, and J. Cai, "Medium access control in ultrawideband wireless networks," IEEE Trans. Veh. Technol., vol. 54, no. 5, pp. 1663-1677, Sept. 2005.

[10] P. Gupta and P. Kumar, "The capacity of wireless networks," IEEE Trans. Inform. Theory, vol. IT-46, pp. 388-404, Mar. 2000.

[11] A. E. Gamal, J. Mammen, B. Prabhakar, and D. Shah, "Optimal throughput-delay scaling in wireless networks-part I: the fluid model," IEEE Trans. Networking, vol. 14, no. SI, pp. 2568-2592, June 2006.

[12] B. Radunovic and J. L. Boudec, "Optimal power control, scheduling, and routing in UWB networks," IEEE J. Select. Areas Commun., vol. 22, no. 7, pp. 1252-1270, Sept. 2004.

[13] L. X. Cai, L. Cai, X. Shen, and J. W. Mark, "Capacity of UWB networks supporting multimedia services," in Proc. IEEE Qshine'06, Aug. 2006.

[14] L. X. Cai, X. Shen, J. W. Mark, and L. Cai, "Capacity analysis and MAC enhancement for UWB broadband wireless access networks," Elsevier Computer Networks, vol. 51, no. 11, pp. 3265-3277, Aug. 2007.

[15] K. Liu, L. Cai, and X. Shen, "Multi-class QoS scheduling in UWB networks using discrete stochastic optimization," IEEE Trans. Veh. Technol., vol. 57, no. 2, pp. 1178-1187, Mar. 2008.

[16] Y. Yi, G. de Veciana, and S. Shakkottai, "On optimal MAC scheduling with physical interference," in Proc. IEEE Infocom'07, May 2007, pp. 294-302.

[17] K. K. P. Chaporkar and S. Sarkar, "Throughput guarantees through maximal scheduling in wireless networks," in Proc. 43rd Annual Allerton Conference on Communication, Control and Computing, 2005.

[18] X. Lin and N. B. Shroff, "The impact of imperfect scheduling on cross-layer rate control in wireless networks," in Proc. IEEE Infocom'05, vol. 3, 2005, pp. 1804-1814. [Online]. Available: http://ieeexplore.ieee.org/xpls/abs_all.jsp?arnumber=1498460

[19] X. Wu, R. Srikant, and R. James, "Scheduling efficiency of distributed greedy scheduling algorithms in wireless networks," IEEE Trans. Mobile Computing, vol. 6, pp. 595-605, June 2007.

[20] R. Merz, J. Widmer, J.-Y. L. Boudec, and B. Radunovic, "A joint PHY/MAC architecture for low-radiated power TH-UWB wireless adhoc networks: research articles," Wirel. Commun. Mob. Comput., no. 5, pp. 567-580, 2005.

[21] B. Hajek, A. Krishna, and R. Lamaire, "On the capture probability for a large number of stations," IEEE Trans. Commun., vol. 45, pp. 254-260, Feb. 1997.

[22] T. Kim, H. Lim, and J. C. Hou, "Improving spatial reuse through tuning transmit power, carrier sense threshold, and data rate in multihop wireless networks," in Proc. MobiCom'06, pp. 366-377.

[23] X. Yang and N. H. Vaidya, "On physical carrier sensing in wireless ad hoc networks," in Proc. IEEE Infocom'05, vol. 4, Mar. 2005, pp. $2525-2535$.

[24] J. Zhu, S. Roy, X. Guo, and W. S. Conner, "Maximizing aggregate throughput in 802.11 mesh networks with physical carrier sensing and two-radio multi-channel clustering," in Proc. NSF-FPI Workshop, 2004. 
[25] D. Jing, B. Liang, and P. K. Varshney, "Tuning the carrier sensing range of IEEE 802.11 MAC,” in Proc. IEEE Globecom'04, vol. 5, Nov. 2004, pp. 2987-2991.

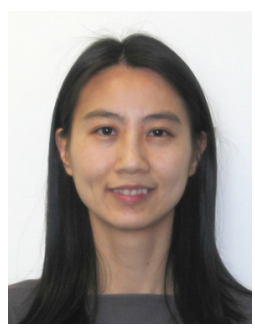

Lin X. Cai received the B.Sc. degree in Computer Science from Nanjing University of Science and Technology, Nanjing, China, in 1996 and the M.A.Sc. degree in Electrical and Computer Engineering from the University of Waterloo, Waterloo, Canada, in 2005. She is currently working toward the Ph.D. degree in the same field at the University of Waterloo. Her current research interests include network performance analysis and protocol design for multimedia applications over broadband wireless access networks.

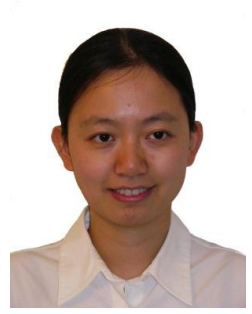

Lin Cai (S'00-M'06) received the M.A.Sc. and $\mathrm{Ph} . \mathrm{D}$. degrees (with Outstanding Achievement in Graduate Studies Award) in electrical and computer engineering from the University of Waterloo, Waterloo, Canada, in 2002 and 2005, respectively. Since July 2005, she has been an Assistant Professor in the Department of Electrical and Computer Engineering at the University of Victoria, British Columbia, Canada. Her research interests span several areas in wireless communications and networking, with a focus on network protocol and architecture design supporting emerging multimedia traffic over wireless, mobile, ad hoc, and sensor networks. She serves as the Associate Editor for IEEE TRANSACTIONS ON VEHICULAR TECHNOLOGY (2007- ), EURASIP JOURNAL ON WIRELESS COMMUNICATIONS AND NETWORKING (2006- ), and INTERNATIONAL JOURNAL OF SENSOR NETWORKS (2006- ).

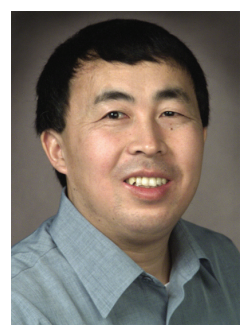

Xuemin (Sherman) Shen (M'97-SM'02-F'09) received the B.Sc.(1982) degree from Dalian Maritime University (China) and the M.Sc. (1987) and Ph.D. degrees (1990) from Rutgers University, New Jersey (USA), all in electrical engineering. $\mathrm{He}$ is a University Research Chair Professor, Department of Electrical and Computer Engineering, University of Waterloo, Canada. His research focuses on mobility and resource management in interconnected wireless/wired networks, UWB wireless communications networks, wireless network security, wireless body area networks and vehicular ad hoc and sensor networks. He is a coauthor of three books, and has published more than 400 papers and book chapters in wireless communications and networks, control and filtering. He serves as the Tutorial Chair for IEEE ICC'08, the Technical Program Committee Chair for IEEE Globecom'07, the General Co-Chair for Chinacom'07 and QShine'06, the Founding Chair for IEEE Communications Society Technical Committee on P2P Communications and Networking. He also serves as a Founding Area Editor for IEEE TRANSACTIONS ON WIRELESS COMMUNiCATIONS; Editor-in-Chief for PEER-TO-PEER NetworkING AND APPLICATION; Associate Editor for IEEE TRANSACTIONS ON VEHICULAR TECHNOLOGY; KICS/IEEE JOURNAL OF COMMUNICATIONS AND Networks, Computer Networks; ACM/Wireless Networks; and Wireless COMMUNiCATIONS AND MOBILE COMPUTING (Wiley), etc. $\mathrm{He}$ has also served as Guest Editor for IEEE JSAC, IEEE WIRELESS COMMUNiCATIONS, IEEE COMMUNICATIONS MAGAZINE, and ACM MoBILE Networks AND ApPliCATIONS, etc. Dr. Shen received the Excellent Graduate Supervision Award in 2006, and the Outstanding Performance Award in 2004 and 2008 from the University of Waterloo, the Premier's Research Excellence Award (PREA) in 2003 from the Province of Ontario, Canada, and the Distinguished Performance Award in 2002 and 2007 from the Faculty of Engineering, University of Waterloo. Dr. Shen is a Fellow of IEEE, and a Distinguished Lecturer of IEEE Communications Society. He is also a registered Professional Engineer of Ontario, Canada.

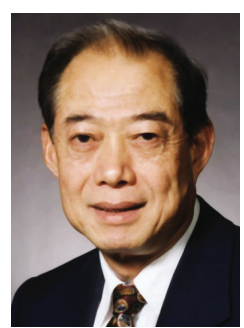

Jon W. Mark (M'62-SM'80-F'88-LF'03) received the B.A.Sc.degree from the University of Toronto in 1962, and the M.Eng. and Ph.D. degrees from McMaster University in 1968 and 1970, respectively, all in electrical engineering. From 1962 to 1970, he was an engineer and then a senior engineer at Canadian Westinghouse Co. Ltd., Hamilton, Ontario, Canada. In September 1970 he joined the Department of Electrical and Computer Engineering, University of Waterloo, Waterloo, Ontario, where he is currently a Distinguished Professor Emeritus. He served as the Department Chairman during the period July 1984-June 1990. In 1996 he established the Centre for Wireless Communications (CWC) at the University of Waterloo and is currently serving as its founding Director. Dr. Mark had been on sabbatical leave at the following places: IBM Thomas J. Watson Research Center, Yorktown Heights, NY, as a Visiting Research Scientist (1976-77); AT\&T Bell Laboratories, Murray Hill, NJ, as a Resident Consultant (1982-83): Laboratoire MASI, Université Pierre et Marie Curie, Paris France, as an Invited Professor (1990-91); and Department of Electrical Engineering, National University of Singapore, as a Visiting Professor (199495). He has previously worked in the areas of adaptive equalization, image and video coding, spread spectrum communications, computer communication networks, ATM switch design and traffic management. His current research interests are in broadband wireless communications and networking, resource and mobility management, and cross domain interworking. He is a co-author of the text entitled Wireless Communications and Networking, Prentice-Hall 2003, and the book entitled Wireless Broadband Networks, to be published by Wiley \& Sons, March 2009. A Fellow of the Canadian Academy of Engineering and a Life Fellow of IEEE, Dr. Mark is the recipient of the 2000 Canadian Award for Telecommunications Research and the 2000 Award of Merit of the Education Foundation of the Federation of Chinese Canadian Professionals. He was an editor of IEEE TRANS ACTIONS ON COMMUNICATIONS (1983-1990), a member of the Inter-Society Steering Committee of the IEEE/ACM TRANSACTIONS ON NETWORKING (1992-2003), a member of the IEEE Communications Society Awards Committee (1995-1998), an editor of WIRELESS NETWORKS (1993-2004), and an associate editor of TELECOMMUNICATION SYSTEMS (1994-2004). He is currently a member of the Advisory Board of the Wiley Series: Advanced Texts in Communications and Networking.

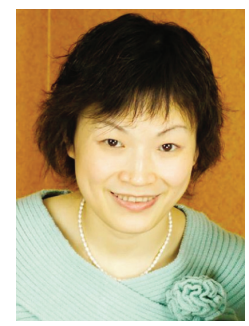

Qian Zhang (M'00-SM'04) received the BS, MS, and $\mathrm{PhD}$ degrees from Wuhan University, China, in 1994, 1996, and 1999, respectively, all in computer science. She joined the Hong Kong University of Science and Technology in September 2005 as an Associate Professor. Before that, she was at Microsoft Research Asia, Beijing, China, from July 1999, where she was the research manager of the Wireless and Networking Group. She has published more than 200 refereed papers in international leading journals and key conferences in the areas of wireless/Internet multimedia networking, wireless communications and networking, and overlay networking. She is the inventor of about 30 pending patents. Her current research interests are in the areas of wireless communications and networking, IP networking, multimedia, $\mathrm{P} 2 \mathrm{P}$ overlay, and wireless security. She has also participated many activities in the IETF ROHC (Robust Header Compression) WG group for TCP/IP header compression. Dr. Zhang is/was the associate editor for the IEEE TRANSACTIONS ON WIRELESS COMMUNICATIONS, IEEE TRANSACTIONS on Multimedia, IEEE TRANSACTIONS ON Vehicular TeChNOLOgY, COMPUTER NETWORKS and COMPUTER COMMUNICATIONS. She has also served as guest editor for the IEEE WIRELESS COMMUNICATIONS, IEEE Journal ON SELECTED AREAS IN COMMUNiCATIONS, IEEE COMMUNICATION MAGAZINE, ACM/SPRINGER Journal of Mobile NeTworks AND APPlications (MONET), and COMPUTER NeTwORKs. She has also involved in the organization committee for many IEEE and ACM conferences. Dr. Zhang received TR 100 (MIT Technology Review) world's top young innovator award in 2004, the Best Asia Pacific (AP) Young Researcher Award elected by the IEEE Communication Society in 2004, and the Best Paper Award by the Multimedia Technical Committee (MMTC) of the IEEE Communications Society and Best Paper Award in QShine 2006, IEEE Globecom 2007 and IEEE ICDCS 2008. She received the Oversea Young Investigator Award from the National Natural Science Foundation of China (NSFC) in 2006. Dr. Zhang is the Chair of the Multimedia Communication Technical Committee of the IEEE Communications Society. 